\title{
A New Type of Distributed Parameter Control Systems: Two-Point Boundary Value Problems for Infinite-Dimensional Dynamical Systems
}

\author{
De-Xing Kong and Fa Wu \\ Department of Mathematics, Zhejiang University, Hangzhou 310027, China \\ Correspondence should be addressed to De-Xing Kong; dkong@zju.edu.cn
}

Received 14 June 2013; Accepted 3 July 2013

Academic Editor: Zhiwei Gao

Copyright (C) 2013 D.-X. Kong and F. Wu. This is an open access article distributed under the Creative Commons Attribution License, which permits unrestricted use, distribution, and reproduction in any medium, provided the original work is properly cited.

This survey note describes a new type of distributed parameter control systems-the two-point boundary value problems for infinite-dimensional dynamical systems, particularly, for hyperbolic systems of partial differential equations of second order, some of the discoveries that have been done about it and some unresolved questions.

\section{A New Type of Distributed Parameter Control Systems: Two-Point Boundary Value Problem for Hyperbolic System of Partial Differential Equations of Second Order}

The mathematical theory of dynamical systems is based on the famous $\mathrm{H}$. Poincarés qualitative theory on ordinary differential equations; the works of A. M. Lyapunov and A. A. Andronov also play an essential role in its development. At present, the theory of dynamical systems is an intensively developing branch of modern mathematics, which is closely connected to the theory of ordinary differential equations. Infinite-dimensional dynamical systems generated by nonlinear evolutionary partial differential equations have been rapidly developing in recent years. In this survey note, we will describe a new type of distributed parameter control systems generated by hyperbolic systems of partial differential equations of second order-the two-point boundary value problems. This kind of problems plays an important role in control theory, mathematics, mechanics, physics, and in other areas of sciences and technology.

It is well known that there are many deep and beautiful results on the TBVPs for ordinary differential equations of second order. However, according to the authors' knowledge, only a few of the results on the TBVPs for hyperbolic equations or other nonlinear evolutionary partial differential equations (even for linear or nonlinear wave equations) have been known.

In 2010, the first author introduced the TBVPs for linear wave equations (see page 121 of [1]). For simplicity, we consider the following wave equation:

$$
u_{t t}-c^{2} \Delta u=0
$$

where $u=u(t, x)$ is the unknown scalar function, $\Delta=$ $\sum_{i=1}^{n}\left(\partial^{2} / \partial x_{i}^{2}\right)$ denotes the Laplacian, and $c$ is a fixed constant which stands for the propagation speed of the wave. The TBVP for (1) reads as follows:

TBVP for (1). Given two functions $f(x)$ and $g(x) \in$ $C^{[n / 2]+2}\left(\mathbb{R}^{n}\right)$ and a positive constant $T$, can we find a $C^{2}$ smooth function $u=u(t, x)$ defined on the strip $[0, T] \times \mathbb{R}^{n}$ such that the function $u=u(t, x)$ satisfies (1) on the domain $[0, T] \times \mathbb{R}^{n}$, the initial condition

$$
u(0, x)=f(x), \quad \forall x \in \mathbb{R}^{n},
$$

and the terminal condition

$$
u(T, x)=g(x), \quad \forall x \in \mathbb{R}^{n} ?
$$


The problem mentioned earlier can be expressed in another way.

Another Statement. Given two functions $f(x)$ and $g(x) \epsilon$ $C^{[n / 2]+2}\left(\mathbb{R}^{n}\right)$ and a positive constant $T$, can we find an initial velocity $v=v(x)$ such that the Cauchy problem for wave equation (1) with the initial data

$$
t=0: \quad u=f(x), \quad u_{t}=v(x),
$$

has a solution $u=(t, x) \in C^{2}\left([0, T] \times \mathbb{R}^{n}\right)$ which satisfies terminal condition (3)?

Moreover, in [1], the author also introduced the TBVPs for nonlinear wave equations including the nonlinear vibrating string and the semilinear wave equation (see page 122 of [1]). Similarly, we can propose the two-point boundary value problems for infinite-dimensional dynamical systems, particularly, the following general (linear or nonlinear) hyperbolic partial differential equation of second order:

$$
\mathscr{P}\left(t, x, u, D u, D^{2} u\right)=0,
$$

where $t$ is the time variable, $x=\left(x_{1}, \ldots, x_{n}\right)$ stand for the spacial variables, $u=u(t, x)$ is the unknown function, and $\mathscr{P}$ is a given smooth function of the independent variables $t, x_{1}, \ldots, x_{n}$, the unknown function $u$, the first-order partial derivatives $D u=\left(u_{t}, u_{x_{1}}, \ldots, u_{x_{n}}\right)$, and the second-order partial derivatives $D^{2} u=\left(u_{t t}, u_{t x_{1}}, \ldots\right)$ (see [2]).

In fact, the system (1) together with conditions (2)-(3) can be viewed as a distributed parameter control system when the initial velocity function $v(x)$ is considered as a control input.

We now introduce the following definition.

Definition 1. For any given $T>0$, if the TBVP (1), (2)-(3) has a $C^{2}$ solution on the strip $[0, T] \times \mathbb{R}^{n}$, then the system (1), (2)(3) is called to possess the global exact controllability. If the TBVP (1), (2)-(3) admits a $C^{2}$ solution on the strip $[0, T] \times \mathbb{R}^{n}$ for some given $T>0$, then one can say that the system (1), (2)-(3) possesses the exact controllability.

Remark 2. For a hyperbolic system of (linear or nonlinear) partial differential equations of second order, we have similar concepts and definitions as mentioned earlier. For hyperbolic partial differential equations of higher order, or more general infinite-dimensional dynamical systems, we also have a similar discussion.

In this survey note, we will describe the two-point boundary value problems for general infinite-dimensional dynamical systems, in particular, hyperbolic system of partial differential equations of second order, some of the discoveries that have been done about it and some unresolved questions.

\section{Known Results}

Since the two-point boundary value problems for (in particular, nonlinear) hyperbolic partial differential equations of second order are too complex and difficult, they are, in our opinion, challenging problems in the field of nonlinear partial differential equations. Up to now, only a few results on the TBVPs for nonlinear hyperbolic equations or other nonlinear evolutionary partial differential equations (even for nonlinear wave equations) have been known. We now recall some known results on the TBVPs for hyperbolic partial differential equations of second order.

Kong and Sun in [2] proved the following result.

Theorem 3. The TBVP (1)-(3) admits a $C^{2}$-smooth solution $u=u(t, x)$ defined on the strip $[0, T] \times \mathbb{R}^{n}$.

This theorem shows that wave equation (1) possesses the global exact controllability. However, the solution of the TBVP of (1)-(3) does not possess the uniqueness (see [2] for the details). On the other hand, by [2], Theorem 3 can be generalized to the case of the following inhomogeneous wave equations: $u_{t t}-c^{2} \Delta u=G(t, x)$, where $G(t, x)$ is a given function which stands for the source term of the system.

Similar results are also true for some nonlinear wave equations including a wave map equation arising from geometry and the equations for the motion of relativistic strings in the Minkowski space-time $\mathbb{R}^{1+n}$ (see [2]). It is well known that these nonlinear wave equations play an important role in both modern mathematics and theoretical physics.

We now consider the The TBVP of wave equation defined on the unit circle:

$$
\begin{gathered}
u_{t t}-u_{\theta \theta}=0, \quad \forall(t, \theta) \in[0, T] \times \mathbb{S}^{1}, \\
u(0, \theta)=f(\theta), \quad \forall \theta \in \mathbb{S}^{1}, \\
u(T, \theta)=g(\theta), \quad \forall \theta \in \mathbb{S}^{1},
\end{gathered}
$$

where $\mathbb{S}^{1}$ stands for the unit circle, $f(\theta)$ and $g(\theta)$ are two given $C^{2}$ functions of $\theta \in \mathbb{S}^{1}$, and their derivative of third order is piecewise smooth. The following result has been proved by [2].

Theorem 4. The TBVP (6) admits a global $C^{2}$ solution $u=$ $u(t, \theta)$ defined on the cylinder $[0, T] \times \mathbb{S}^{1}$, provided that $T / 2 \pi$ is a rational number and $T / \pi \notin \mathbb{N}$.

Theorem 4 implies that the wave equation defined on a circle possesses the exact controllability. Moreover, for the $(1+n)$-dimensional wave equation (1), we have similar results, provided that the initial/terminal data $f(x)$ and $g(x)$ in (2)(3) are suitable smooth functions and are periodic in $r=$ $\sqrt{x_{1}^{2}+\cdots+x_{n}^{2}}$.

As an application of the results mentioned earlier, Kong and Sun in [2] introduced a hyperbolic curvature flow and obtained a result analogous to the well-known theorem of Gage and Hamilton [3] for the curvature flow of plane curves.

\section{Open Problems}

Although we have proved the existence of solutions to the TBVPs for linear wave equations or some special nonlinear wave equations, there are quite a number of problems here. 
In particular, the following open problems seem to be more interesting and important.

Problem 1. The two-point boundary value problem for a semilinear wave equation

$$
u_{t t}-u_{x x}=f(u)
$$

with a regular and bounded function $f(y)$. For example, $f(u)=\sin u$ corresponds the famous sine-Gorden equation.

Problem 2. The two-point boundary value problem for a quasilinear wave equation

$$
u_{t t}-\left(\mathscr{C}\left(u_{x}\right)\right)_{x}=0
$$

with a smooth and increasing function $\mathscr{C}(\nu)$, for example, $\mathscr{C}(\nu)=v / \sqrt{1+v^{2}}$, which describes the nonlinear vibrating string.

Problem 3. The two-point boundary value problem for the following hyperbolic Monge-Ampère equation (see [4])

$$
S_{\tau \tau}=\frac{S_{\theta \tau}^{2}-1}{S_{\theta \theta}+S}, \quad \forall(\theta, \tau) \in \mathbb{S}^{1} \times[0, T) .
$$

This problem plays an important role in geometric analysis, crystal growth, and so forth. Precisely speaking, if does there exist a smooth solution $S$ to (9) such that the following conditions hold

$$
S(0, \theta)=S_{0}(\theta), \quad S(T, \theta)=S_{T}(\theta) ?
$$

where $S_{0}(\theta)$ and $S_{T}(\theta)$ are two smooth and positive functions defined on $\mathbb{S}^{1}$.

Problem 4. Two-point boundary value problems for multidimensional version of the equations mentioned above. Moreover, it is worthy to investigate the two-point boundary value problems for the systems of multidimensional partial differential equations, for example, Einstein's field equations and the system of time-like extremal submanifolds in physical space-times and so forth.

Problem 5. The two-point boundary value problem for hyperbolic geometric flow: Let $\mathscr{M}$ be $n$-dimensional complete compact Riemannian manifold with the Riemannian metric $g_{0}$. Given a positive constant $T$ and another Riemannian metric $g_{T}$ on $\mathscr{M}$, if does there exist a smooth solution $g$ to the hyperbolic geometric flow equation

$$
\frac{\partial^{2} g_{i j}}{\partial t^{2}}=-2 R_{i j}
$$

such that the following conditions hold

$$
g(0)=g_{0}, \quad g(T)=g_{T} ?
$$

Obviously, (11) is a nonlinear system of second-order partial differential equations for a family of Riemannian metrics $g_{i j}(t)$ on $\mathscr{M}$.
Problem 6. Two-point boundary value problems for infinitedimensional dynamical systems in the framework of weak solutions. For example, we consider the following two-point boundary value problem:

$$
\begin{gathered}
\mathbf{r}_{t t}-\mathbf{r}_{\theta \theta}=0, \quad \forall(t, \theta) \in[0, T] \times \mathbb{S}^{1}, \\
\mathbf{r}(0, \theta)=\mathbb{O}(\theta), \quad \forall \theta \in \mathbb{S}^{1}, \\
\mathbf{r}(T, \theta)=\mathbb{T}(\theta), \quad \forall \theta \in \mathbb{S}^{1},
\end{gathered}
$$

where $\mathbf{r}=\left(r_{1}(t, \theta), r_{2}(t, \theta)\right)$ is the unknown vector-valued function and $\mathbb{O}(\theta)$ and $\mathbb{T}(\theta)$ stand for a circle and a square, respectively. How about the existence and uniqueness of the weak solution? These are interesting problems worthy to study, in particular, for nonlinear partial differential equations.

\section{Acknowledgments}

This work was supported in part by the NNSF of China (Grant no. 11271323) and by Zhejiang Provincial Natural Science Foundation of China (Grant no. LZ13A010002).

\section{References}

[1] D.-X. Kong, Partial Differential Equations, Higher Education Press, Beijing, China, 2010.

[2] D.-X. Kong and Q.-Y. Sun, "Two-point boundary value problems and exact controllability for several kinds of linear and nonlinear wave equations," Journal of Physics, vol. 290, article 012008, 26 pages, 2011.

[3] M. Gage and R. S. Hamilton, "The heat equation shrinking convex plane curves," Journal of Differential Geometry, vol. 23, no. 1, pp. 69-96, 1986.

[4] D.-X. Kong, K. Liu, and Z. Wang, "Hyperbolic mean curvature flow: evolution of plane curves," Acta Mathematica Scientia, vol. 29, no. 3, pp. 493-514, 2009. 


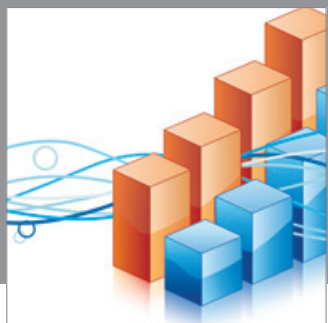

Advances in

Operations Research

mansans

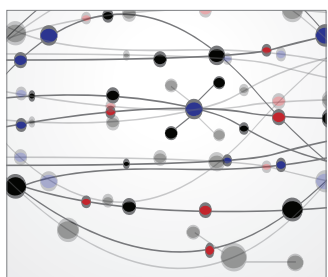

The Scientific World Journal
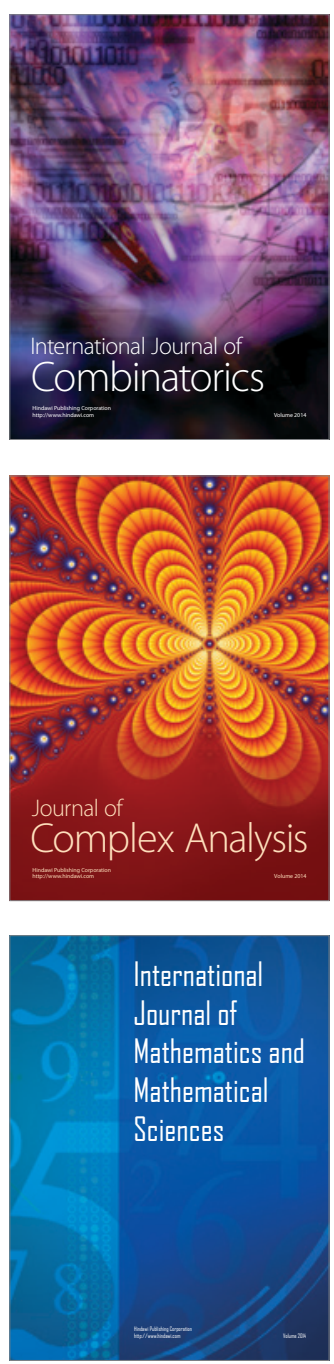
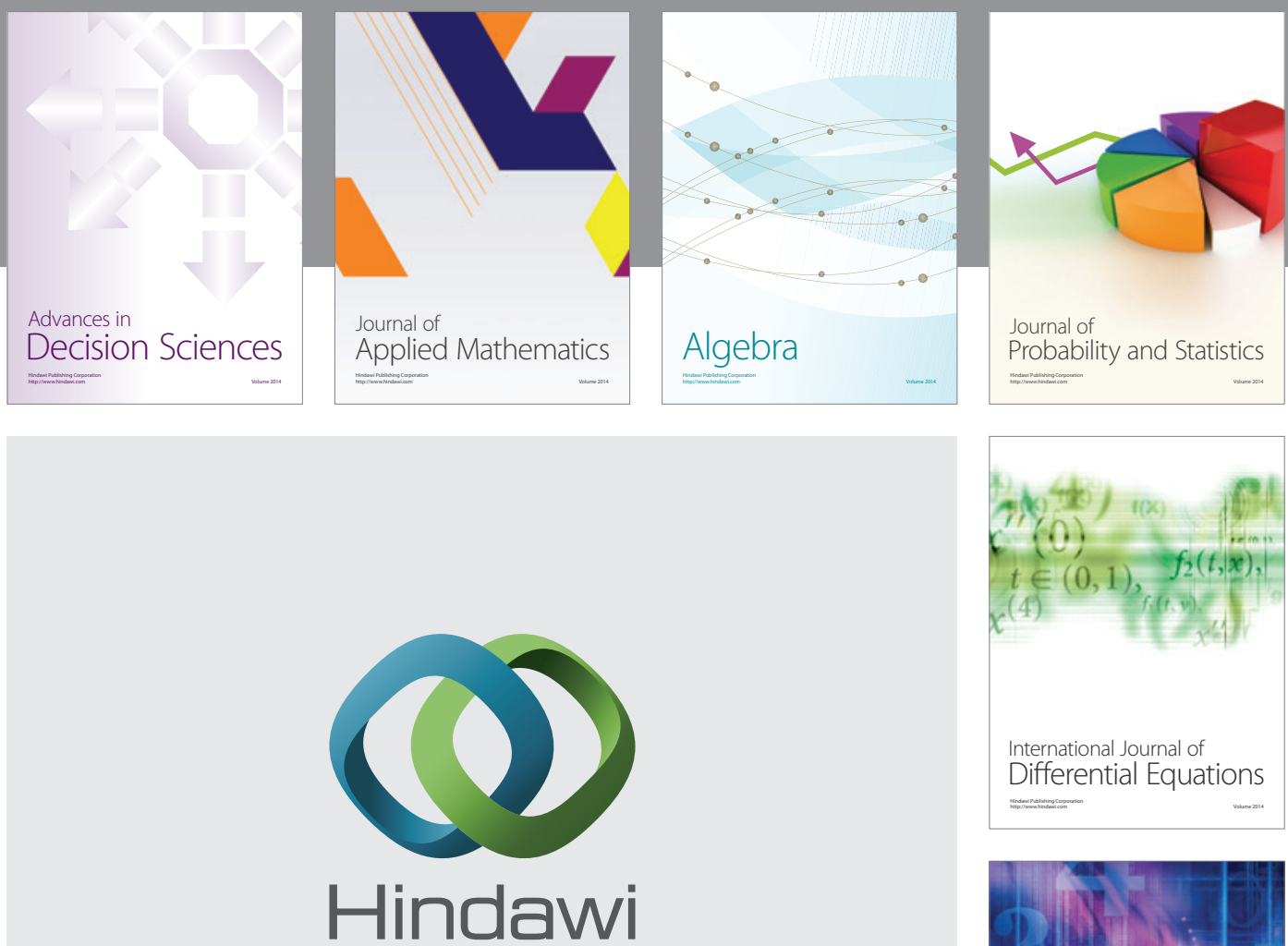

Submit your manuscripts at http://www.hindawi.com
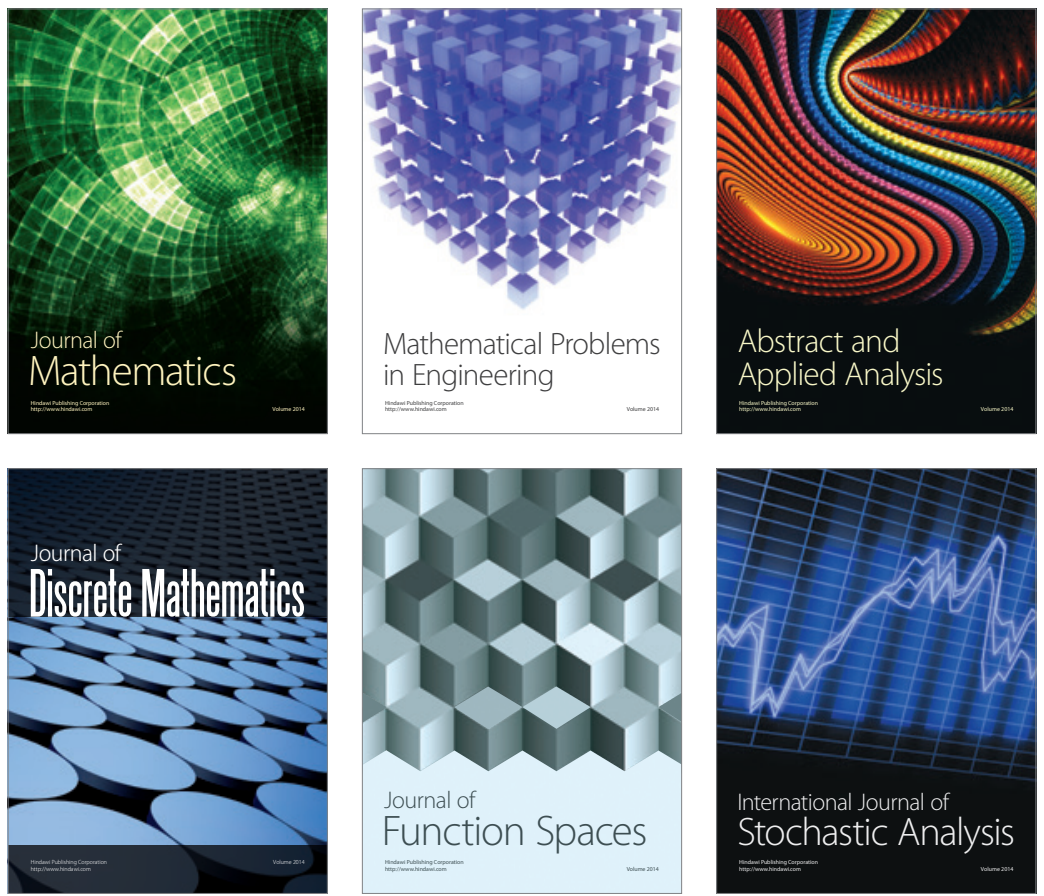

Journal of

Function Spaces

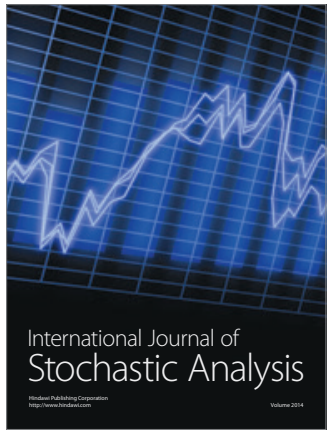

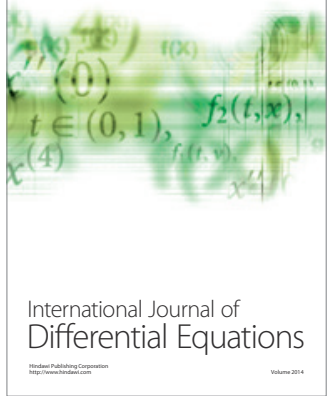
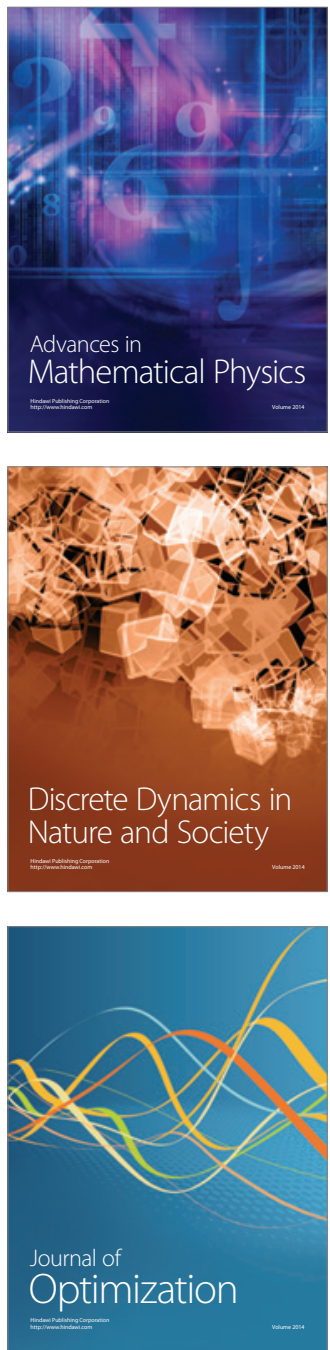